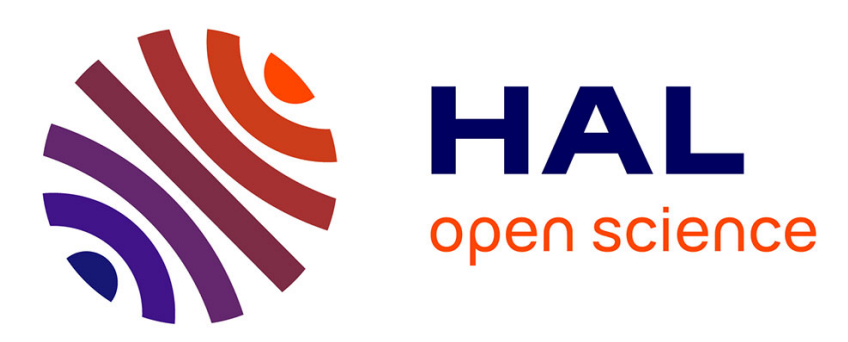

\title{
Analytical prediction of the hydraulic jump detachment length in front of mounted obstacles in supercritical open-channel flows
}

Lorris Gond, Gaële Perret, Emmanuel Mignot, Nicolas Rivière

\section{- To cite this version:}

Lorris Gond, Gaële Perret, Emmanuel Mignot, Nicolas Rivière. Analytical prediction of the hydraulic jump detachment length in front of mounted obstacles in supercritical open-channel flows. Physics of Fluids, 2019, 31 (4), pp.045101. 10.1063/1.5085744 . hal-02381377

\section{HAL Id: hal-02381377 \\ https://hal.science/hal-02381377}

Submitted on 26 Nov 2019

HAL is a multi-disciplinary open access archive for the deposit and dissemination of scientific research documents, whether they are published or not. The documents may come from teaching and research institutions in France or abroad, or from public or private research centers.
L'archive ouverte pluridisciplinaire HAL, est destinée au dépôt et à la diffusion de documents scientifiques de niveau recherche, publiés ou non, émanant des établissements d'enseignement et de recherche français ou étrangers, des laboratoires publics ou privés. 


\section{Analytical prediction of the hydraulic jump detachment length in front of mounted obstacles in supercritical open-channel flows}

Cite as: Phys. Fluids 31, 045101 (2019); https://doi.org/10.1063/1.5085744

Submitted: 14 December 2018 . Accepted: 12 March 2019 . Published Online: 02 April 2019

Lorris Gond, Gaele Perret, Emmanuel Mignot, and Nicolas Riviere (D)
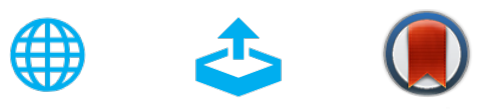

\section{ARTICLES YOU MAY BE INTERESTED IN}

Construction of knotted vortex tubes with the writhe-dependent helicity

Physics of Fluids 31, 047101 (2019); https://doi.org/10.1063/1.5088015

Coexistence of acoustic waves and turbulence in low Mach number compressible flows Physics of Fluids 31, 045102 (2019); https://doi.org/10.1063/1.5087056

Dynamic mode decomposition for the inspection of three-regime separated transitional boundary layers using a least squares method

Physics of Fluids 31, 044103 (2019); https://doi.org/10.1063/1.5086225

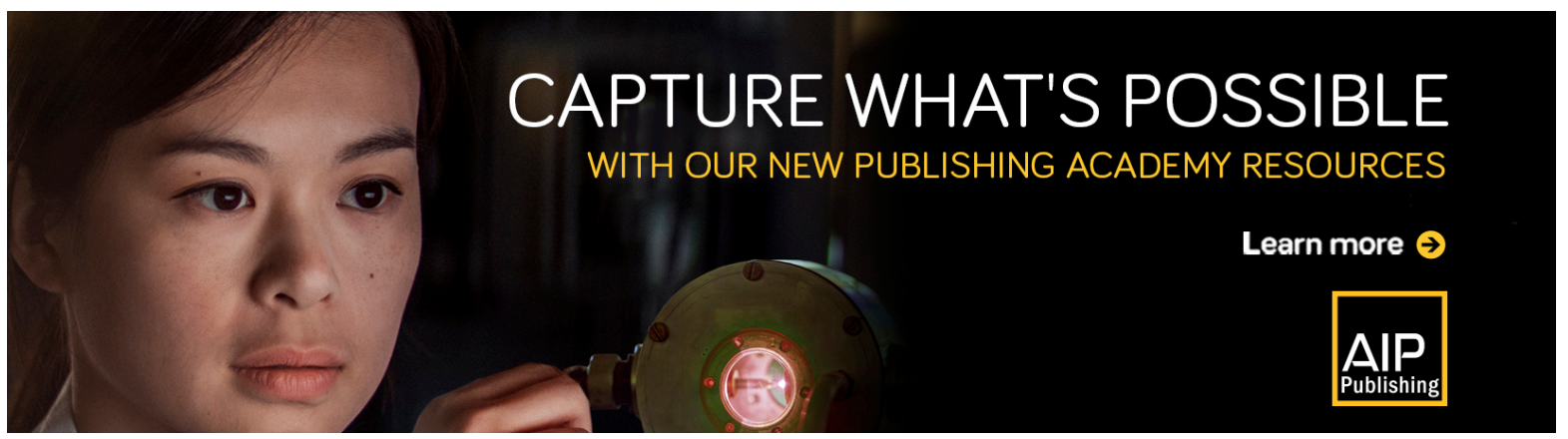




\title{
Analytical prediction of the hydraulic jump detachment length in front of mounted obstacles in supercritical open-channel flows
}

\author{
Cite as: Phys. Fluids 31, 045101 (2019); doi: 10.1063/1.5085744 \\ Submitted: 14 December 2018 - Accepted: 12 March 2019 • \\ Published Online: 2 April 2019
}

Lorris Gond, ${ }^{1,2}$ Gaele Perret, ${ }^{2}$ Emmanuel Mignot, ${ }^{1, a)}$ and Nicolas Riviere

\begin{abstract}
AFFILIATIONS
${ }^{1}$ Université Lyon, INSA Lyon, CNRS, LMFA UMR5509, F-69621 Villeurbanne, France

${ }^{2}$ Université Le Havre Normandie, CNRS, LOMC UMR, 6294 Le Havre, France
\end{abstract}

a) Author to whom correspondence should be addressed: Emmanuel.mignot@insa-lyon.fr

\begin{abstract}
Detached hydraulic jumps are major features of supercritical open-channel flows interacting with emerging obstacles. Such a flow pattern exhibits strong similarities with shock waves detached in front of bluff bodies in supersonic aerodynamic flows. This paper aims at evaluating the capacities of an analytical model, adapted from supersonic aerodynamics, to predict the hydraulic jump detachment length. The analytical predictions are compared to the measured hydraulic jumps from two experiments: (i) a uniform supercritical open-channel flow that skirts a mounted and emerging obstacle (with a horseshoe vortex) and (ii) a mounted and emerging obstacle moving at constant velocity in water at rest (without a horseshoe vortex). Moreover, numerical calculations of supercritical flow skirting emerging obstacles are undertaken, with a free-slip condition at the bed to remove the horseshoe vortex, while keeping the detached hydraulic jump. The comparison of the detachment lengths of these experimental, analytical, and computed hydraulic jumps reveals that two types of detachment lengths can be defined. The detachment length visible on experiments corresponds to the toe of the hydraulic jump, while the detachment length predicted by the analytical model rather corresponds to the location of flow regime transition from the supercritical to subcritical regime. The present work thus validates the analytical model for predicting the location of flow regime transition (for configurations without a horseshoe vortex) but not for predicting the toe of the hydraulic jump. We finally confirm the strong connections between two distinct phenomena: a hydraulic jump in water flow and a shock wave in gas flow.
\end{abstract}

Published under license by AIP Publishing. https://doi.org/10.1063/1.5085744

\section{INTRODUCTION}

Supercritical open-channel flows interacting with mounted obstacles are common flow features in regions with steep slopes. During urban floods, due to the limited bottom roughness, the flow in the streets along the main slope can become supercritical (Mignot et al., 2006 and Sturm et al., 2018) and interact with emerging urban furniture such as trees, bus-stops and piles. In mountain areas, the flow regime in rivers can also become supercritical, particularly following heavy rain conditions, and interact with bridge piles. Fishways along dams are often steep and are sometimes covered by impervious blocks that the flow must skirt (Cassan et al., 2014). Jiang and Smith (2000) studied a supercritical free surface shallow water flow over an isolated bump. Finally following tsunamis, the inland flow reaches a supercritical regime and interacts with artificial (building, cars, etc.) and natural (hills, etc.) emerging obstacles (Lukkunaprasit et al., 2009 and Wüthrich et al., 2018). All these obstacles can then be seen as impervious obstacles mounted on the bottom and emerging from the free-surface that the supercritical inflow must skirt it on the sides. In such conditions, Riviere et al. (2017) recently showed that two flow patterns can be observed as a function of the Froude number and the ratio between the incoming water depth and the obstacle typical size. For narrow obstacles or large water depths, a so-called "bow wave like wall jet" forms along the upstream face of the obstacle so that the flow skirts the obstacle as a vertical jet above the water depth. Oppositely, for wider obstacles or smaller water depths, a detached hydraulic jump takes place upstream and on the sides of the obstacle. This detached hydraulic jump was qualitatively described for a rectangular obstacle by Defina and Susin (2006), and its detachment length was measured 


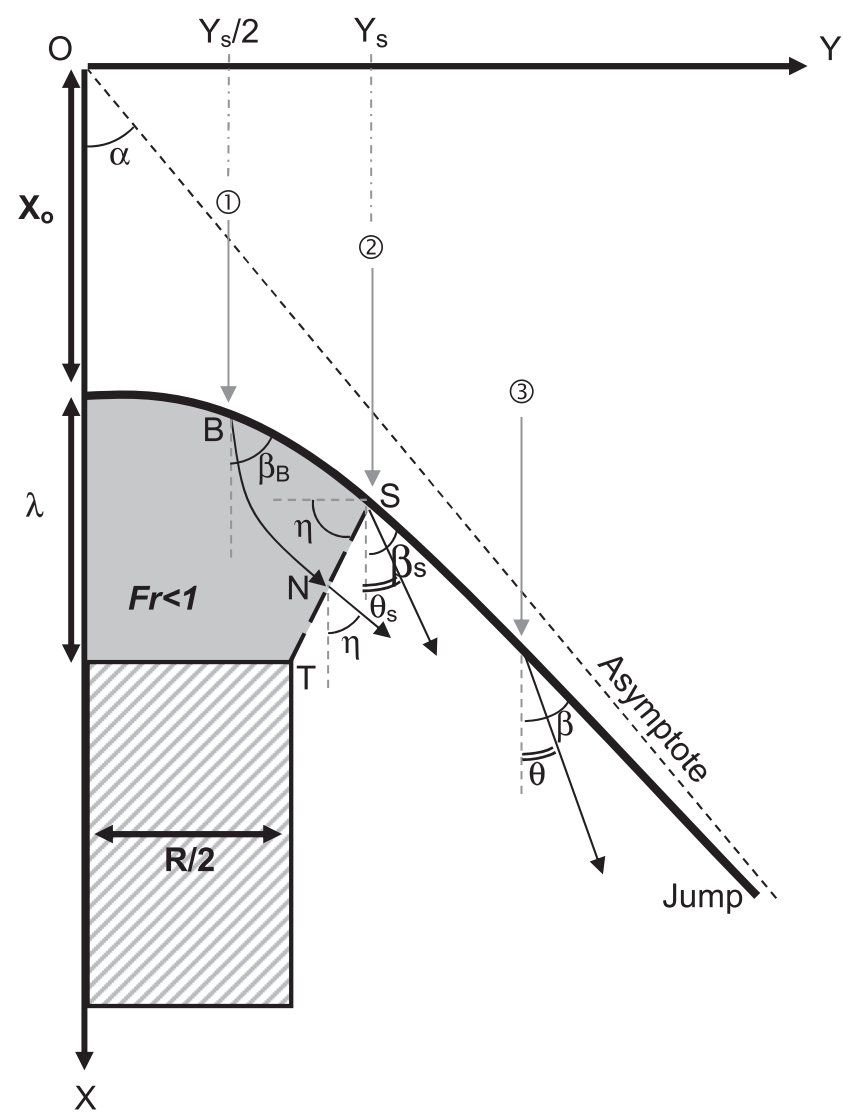

FIG. 1. Top-view sketch of the flow around the obstacle with OX being the symmetry plane of the incoming flow and of the obstacle. Three streamlines are depicted: streamline (1) is the barycentric streamline entering the subcritical zone at point $\mathrm{B}$ and leaving it by crossing the critical line at point N; streamline (2) crosses the hydraulic jump at point $S$ (intersection of the critical line and the hydraulic jump); and streamline (3) crosses the hydraulic jump further away from the obstacle region and thus remains in the supercritical regime. by Mignot and Riviere (2010) for smooth and fixed bed conditions. Mignot and Riviere (2010) additionally revealed the presence of a horseshoe vortex in the near bed region at the foot of the obstacle where the flow regime is subcritical. Recently, Wüthrich et al. (2018) observed the transient transition between the wall jet and detached jumps around obstacles within transient flows reproducing tsunami waves.

Research studies regarding hydraulic jump processes are still going on, including velocity field (Misra et al., 2008) and air entrainment (Witt et al., 2018) measurements and calculations. However, these studies mainly concentrate on straight hydraulic jumps and thus differ from the present flow configuration. Through a dimensional analysis, Mignot and Riviere (2010) showed that three nondimensional parameters govern the detachment length (noted $\lambda$, see Fig. 1) of the hydraulic jump in the symmetry plane upstream from the rectangular obstacle: the Reynolds number $\operatorname{Re}_{\mathrm{h}}=4 U_{1} h_{1} / v$, the Froude number $\mathrm{Fr}_{1}=U_{1} /\left(g h_{1}\right)^{0.5}$, and the normalized water depth $h_{1} / R$, with $U_{1}$ and $h_{1}$, respectively, being the inflow bulk velocity and water depth of the uniform supercritical inflow, $R$ being the obstacle width (in a direction perpendicular to the inflow), and $v$ and $g$ being the water kinematic viscosity and gravity acceleration

$$
\frac{\lambda}{R}=f\left(\operatorname{Fr}_{1}, \operatorname{Re}_{h}, \frac{h_{1}}{R}\right) .
$$

These authors additionally showed that $\lambda$ is unaffected by the Reynolds number, slightly decreases as the Froude number increases, and rapidly increases with the normalized water depth (see Fig. 2). Mignot et al. (2016) also revealed that this detachment length is unaffected by an increase in the bottom roughness coefficient but is strongly affected by the scour and deposition pattern around the obstacle in the case of a mobile bed.

Nevertheless, to the authors' knowledge, no analytical method was proposed to predict this detachment length $\lambda$. The aim of the present work is then to adapt an analytical model proposed by Moeckel (1949) to estimate the detachment length of a shock wave upstream from a supersonic bluff body to the present hydraulic configuration. When comparing the model's predictions with
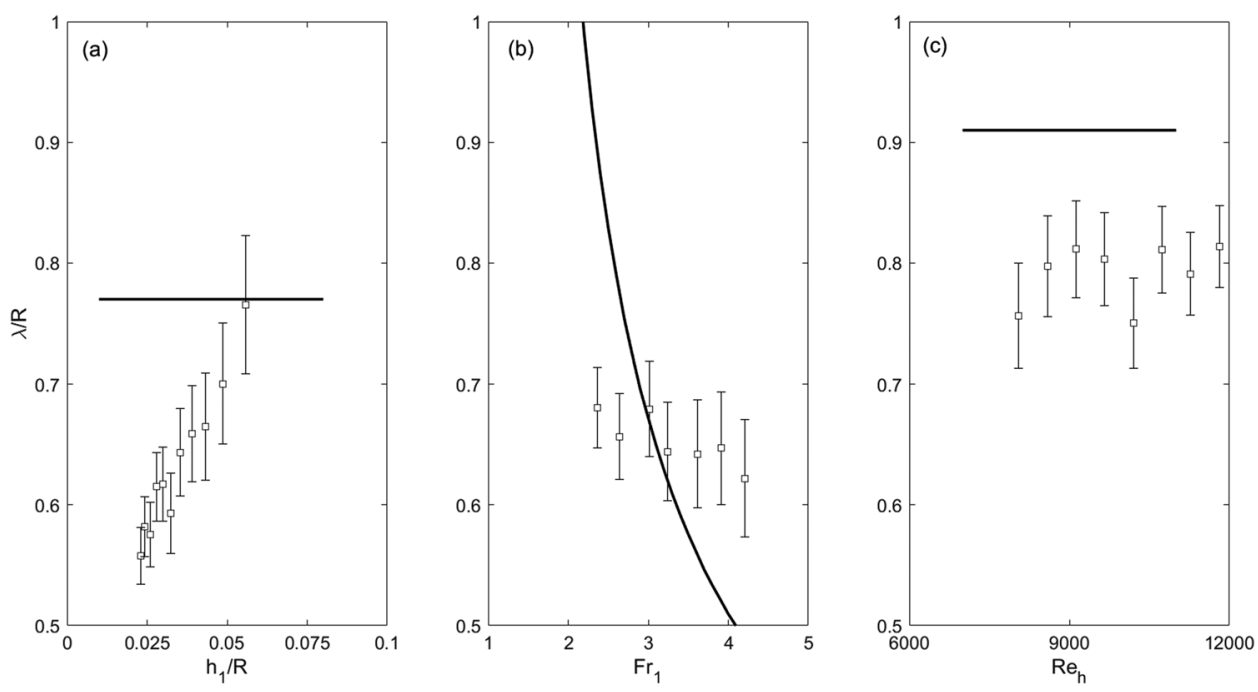

FIG. 2. Comparison of separation distance $\lambda$ measured upstream from the fixed obstacle in supercritical flow at LMFA by Mignot and Riviere (2010) (symbol) and predicted by the analytical model (plain line) as a function of: (a) $h_{1} / R$ (with $\mathrm{Fr}_{1}=2.6$ and $\mathrm{Re}_{\mathrm{h}}=8000$ ), (b) $\mathrm{Fr}_{1}$ (with $h_{1} / R=0.035$ and $\operatorname{Re}_{\mathrm{h}}=8000$ ), and (c) $\operatorname{Re}_{\mathrm{h}}$ (with $h_{1} / R=0.046$ and $\mathrm{Fr}_{1}=2.3$ ). 
experimental aerodynamics measurements, Moeckel (1949) proved the high accuracy of this model. Considering that a shock-wave in aerodynamics is analogous to a hydraulic jump in hydraulics [see, for example, the work of De Chant and Caton (1998) for more information on such analogy], we aim at verifying if this model could predict the detachment length of the hydraulic jump.

Section II is devoted to the adaptation of the model of Moeckel (1949) to a hydraulic configuration and to compare its hydraulic jump detachment length prediction with the data of Mignot and Riviere (2010). Given the poor agreement between the experimental and analytical data, Sec. III introduces a novel experiment with a mounted obstacle moving at constant velocity in water at rest to suppress the horseshoe vortex. Section IV introduces a second approach corresponding to numerical calculations of a supercritical flow around a fixed obstacle, with a free-slip condition at the bed permitting to remove the horseshoe vortex. In Sec. V, the comparison of the hydraulic jump detachment length from the novel experimental approach, the numerical calculation, and the analytical model finally permits to conclude on the capacity of the analytical approach to predict the hydraulic jump detachment length.

\section{ANALYTICAL MODEL}

Consider a supercritical inflow along the $X$ axis skirting a rectangular obstacle of half width $R / 2$ (along the $Y$ axis) and corner $T$, forming a detached hydraulic jump as plotted in Fig. 1. The analytical model, adapted to hydraulics, is based on a first hypothesis introduced by Moeckel (1949) that the shape of the hydraulic jump skirting the obstacle is a hyperbola. Its tangent at $Y=0$ is parallel to the upstream face of the obstacle at the symmetry plane and forms an angle $\alpha=\arctan \left(1 / \sqrt{\mathrm{Fr}_{1}^{2}-1}\right)=\arcsin \left(1 / \mathrm{Fr}_{1}\right)$ with the inflow axis $X$ far away on the sides of the obstacle (see Fig. 1). This geometry, faithful for detached shock waves, was confirmed for detached jumps by Mignot and Riviere (2010). Moeckel's second hypothesis is that the flow is $2 \mathrm{D}$, which is turned here in negligible vertical velocities: the flow can be depth averaged and the hydrostatic pressure assumption holds. Third hypothesis is that the region with the subcritical flow regime (shown in gray in Fig. 1) is delimited, on its sides, by a straight line $T S$ where the flow regime is critical $(\mathrm{Fr}=1)$ with $S$ being the intersection of the critical line TS with the hydraulic jump (location of $S$ is unknown a priori). This critical line forms an angle with the upstream face of the obstacle noted $\eta$ in the following.

\section{A. Geometrical considerations}

In the frame axis $(O, X, Y)$, with the location of $O$ being unknown $a$ priori, the equation of the hyperbola reads

$$
Y^{2}=\frac{X^{2}-X_{0}^{2}}{M^{2}}
$$

where $M=\sqrt{\mathrm{Fr}^{2}-1}$ and $X_{0}$ is the distance between $O$ and the detached jump along the symmetry plane. Deriving Eq. (2) with regard to $X$ reads

$$
\frac{d Y}{d X}=\frac{X}{M \sqrt{X^{2}-X_{0}^{2}}}=\frac{\sqrt{M^{2} Y^{2}+X_{0}^{2}}}{M^{2} Y}=\tan (\beta),
$$

with $\beta$ being the local angle between the detached jump and $X$ axis, so that

$$
\left\{\begin{array}{c}
Y=\frac{X_{0}}{M \sqrt{M^{2} \tan (\beta)^{2}-1}}, \\
X=\frac{M X_{0} \tan (\beta)}{\sqrt{M^{2} \tan (\beta)^{2}-1}}=Y M^{2} \tan (\beta) .
\end{array}\right.
$$

As $\lambda=X_{T}-X_{0}$ and $X_{T}=X_{S}+\left(Y_{S}-R / 2\right) \tan (\eta)$, with $\left(X_{T}, R / 2\right)$ and $\left(X_{S}, Y_{S}\right)$ being the coordinates of points $T$ and $S$, respectively, the detachment length reads

$$
\lambda=X_{S}+\left(Y_{S}-R / 2\right) \tan (\eta)-X_{0} .
$$

Using Eq. (4), Eq. (5) finally reads

$$
\frac{\lambda}{R}=\frac{Y_{S}}{R}\left[M^{2} \tan \left(\beta_{S}\right)-M \sqrt{M^{2} \tan \left(\beta_{S}\right)^{2}-1}+\tan (\eta)\right]-\frac{\tan (\eta)}{2},
$$

where angle $\eta$ can be approximated by

$$
\eta \approx \frac{\theta_{S}-\theta_{\mathrm{MAX}}}{2}
$$

with $\theta_{\mathrm{S}}$ being the deflection angle of streamline (2) when crossing the hydraulic jump at point $S$ (Fig. 1) and $\theta_{\text {MAX }}$ being the maximum deflection angle allowing an oblique jump, which value is given by the formulas of Ippen (1951). For a given inflow $\left(h_{1}, U_{1}\right)$ and obstacle width $(R)$, Eq. (6) then permits to compute $\lambda$ as a function of three unknowns: $Y_{S}, \beta_{\mathrm{S}}$, and $\theta_{\mathrm{S}}$.

\section{B. Mass conservation}

The next step is the application of the mass conservation principle between the supercritical region upstream from the jump (for 0 $<Y<Y_{S}$ ) and line TS where the Froude number equals unity so that the water depth and velocity are critical: $h=h_{C}$ and $U=U_{C}$. This reads

$$
h_{1} U_{1} Y_{S}=\frac{Y_{S}-R / 2}{\cos (\eta)} h_{C} U_{C}
$$

As $\operatorname{Fr}_{\mathrm{C}}=1$, one can write

$$
\frac{h_{1} U_{1}}{h_{C} U_{C}}=\operatorname{Fr}_{1}\left(\frac{h_{1}}{h_{C}}\right)^{\frac{3}{2}}
$$

so that Eq. (8) alternatively reads

$$
\frac{Y_{S}}{R}=\frac{1 / 2}{1-\cos (\eta) \operatorname{Fr}_{1}\left(\frac{h_{1}}{h_{C}}\right)^{\frac{3}{2}}}
$$

$Y_{S} / R$ in Eq. (10) can then be included in Eq. (6) where the three remaining unknowns are $h_{C}, \beta_{S}$, and $\theta_{S}$.

\section{Head conservation}

The subcritical flow approaching the obstacle converges toward the critical line TS, which is a minimum section. It is reasonable, following the model of Moeckel (1949), to assume (i) that the specific head and the water depth are constant along the critical line TS and (ii) that, neglecting head losses downstream the jump, the mean 
specific head along TS equals the average specific head downstream the hydraulic jump. Moeckel (1949) considered, moreover, (iii) that this mean specific head along TS is accurately given by the head on the barycentric streamline (1) at point $B$ located immediately downstream from the detached jump. We verified that integrating numerically the downstream specific head all along the jump confirms this last assumption (not shown here). These three assumptions added to the fact that $\mathrm{Fr}=1$ and the water depth is critical along the critical line TS $\left(h=h_{c}\right)$ lead to

$$
h_{B}+\frac{U_{B}^{2}}{2 g}=h_{C}+\frac{U_{C}^{2}}{2 g}=\frac{2 h_{C}}{3}
$$

so that

$$
\frac{h_{B}}{h_{C}}=\frac{3}{2+\mathrm{Fr}_{B}^{2}} \text {. }
$$

On the other hand, the relation of Ippen (1951) for conjugated water depths across the oblique jump along the barycentric line (at point $B$ ) reads

$$
\frac{h_{1}}{h_{B}}=\frac{2}{\sqrt{1+8\left(\mathrm{Fr}_{1}^{2} \sin \left(\beta_{B}\right)\right)}-1} .
$$

In Eq. (13), one can obtain $\beta_{B}$ as a function of $\beta_{S}$ and $Y_{S}$ using Eq. (4) and the fact that $Y_{B}=Y_{S} / 2$ with

$$
\tan \left(\beta_{B}\right)^{2}=4 \tan \left(\beta_{S}\right)^{2}-\frac{3}{M^{2}} .
$$

Combining Eqs. (12) and (13), one can then write

$$
\frac{h_{1}}{h_{C}}=\frac{h_{1}}{h_{B}} \frac{h_{B}}{h_{C}}=\frac{6}{\left(2+\mathrm{Fr}_{B}^{2}\right)\left[\sqrt{1+8\left(\mathrm{Fr}_{1}^{2} \sin \left(\beta_{B}\right)\right)}-1\right]} \text {, }
$$

with $\beta_{\mathrm{B}}$ being estimated using Eq. (14) as a function of $\beta_{\mathrm{S}}$. When including $h_{C}$ [from Eq. (15)] in Eq. (10) [itself included in Eq. (6)], the three remaining unknowns are now $\beta_{\mathrm{S}}$ and $\theta_{\mathrm{S}}$ and $\mathrm{Fr}_{\mathrm{B}}$.

\section{Determination of deflection and jump angles at point $S$}

According to the relations of Ippen (1951), $\beta$ can be written as a function of the conjugated flow conditions upstream and downstream from the jump by applying a momentum balance along the direction normal to the jump. When applied at point $S$, it reads

$$
\left\{\begin{array}{c}
\beta_{S}=\sin ^{-1}\left(\sqrt{\left.\frac{4\left(\frac{1}{2}+\frac{h_{S}}{h_{1}}\right)^{2}-1}{8 \mathrm{Fr}_{1}^{2}}\right)},\right. \\
\theta_{S}=\beta_{S}-\tan ^{-1}\left(\frac{h_{1}}{h_{S}} \tan \left(\beta_{S}\right)\right) .
\end{array}\right.
$$

Including Eq. (16) in Eq. (15) permits to reduce the number of unknowns to $2: h_{S}$ (the water depth downstream from the jump at point $S$ ) and $\mathrm{Fr}_{\mathrm{B}}$ (the Froude number at point $B$ downstream from the jump along the barycentric line).

According to Ippen (1951), the tangential component of the velocity (parallel to the detached jump locally) remains unchanged on both sides of the oblique jump: $U_{t 1}=U_{t 2}$, which reads

$$
\frac{\mathrm{Fr}_{1} \sqrt{g h_{1}} \sin (\beta)}{\tan (\beta)}=\frac{\mathrm{Fr}_{2} \sqrt{g h_{2}} \sin (\beta-\theta)}{\tan (\beta-\theta)}
$$

so that

$$
\begin{aligned}
& \operatorname{Fr}_{1}^{2} g h_{1} \sin ^{2}(\beta)\left[\frac{1}{\sin ^{2}(\beta)}-1\right] \\
& \quad=\operatorname{Fr}_{2}^{2} g h_{2} \sin ^{2}(\beta-\theta)\left[\frac{1}{\sin ^{2}(\beta-\theta)}-1\right],
\end{aligned}
$$

and finally

$$
\operatorname{Fr}_{1}^{2} g h_{1}\left(1-\sin ^{2}(\beta)\right)=\operatorname{Fr}_{2}^{2} g h_{2}-\operatorname{Fr}_{1}^{2} g h_{1} \sin ^{2}(\beta)\left(\frac{h_{1}}{h_{2}}\right)^{2},
$$

with indices 1 and 2 referring to the region just upstream and downstream from the oblique jump, respectively. Using Eq. (16) (replacing, for the sake of generality, $h_{S}$ by $h_{2}$ the water depth at any location downstream from the jump), Eq. (19) reads

$$
\mathrm{Fr}_{2}^{2}=\frac{h_{1}}{h_{2}}\left[\operatorname{Fr}_{1}^{2}-\frac{1}{2} \frac{h_{1}}{h_{2}}\left(\frac{h_{2}}{h_{1}}-1\right)\left(\frac{h_{2}}{h_{1}}+1\right)^{2}\right] \text {. }
$$

Equation (20) permits to estimate the two remaining unknowns: (i) $\mathrm{Fr}_{2}=\mathrm{Fr}_{\mathrm{B}}$ when setting $h_{2}=h_{B}$ and (ii) $h_{2}=h_{S}$ when setting $\mathrm{Fr}_{2 S}=1$ as point $S$ belongs to the critical line.

Finally, Eq. (6) permits to predict $\lambda$, the detachment length of the hydraulic jump along the symmetry axis.

\section{E. Analytical vs experimental detachment length}

Figure 2 reveals that the normalized detachment length of the hydraulic jump along the symmetry axis predicted by the analytical model only depends on the incoming Froude number $\lambda / R=\mathrm{f}\left(\mathrm{Fr}_{1}\right)$. Figure 2 then compares the detachment lengths measured by Mignot and Riviere (2010) and estimated by the analytical model. While the orders of magnitude are similar for the measurements and predictions, the quantitative agreement is poor:

- The measured detachment length strongly depends on the normalized water depth, while the analytical model does not predict any influence of this parameter [Fig. 2(a)].

- The measurements exhibit a much smaller dependency with regard to the Froude number than the analytical model [Fig. 2(b)].

- Both do not exhibit any dependency on the Reynolds number, but a constant shift is observed in Fig. 2(c).

The discrepancies between the hydraulic jump detachment length measured by Mignot and Riviere (2010) and predicted by the analytical model are expected to be related to one (or both) of the following potential reasons:

- Potential reason \#1 (PR1). The analytical model predicts the location of the transition from the supercritical to subcritical flow regime. Oppositely, Mignot and Riviere (2010) measured the location of the toe of the hydraulic jump (location where the flow starts to be affected by the obstacle and the water depth starts increasing). Unlike for shock waves, hydraulic jumps cannot be considered as infinitely thin so that these two locations should differ. Measured and predicted detachment lengths may not be comparable at all.

- Potential reason \#2 (PR2). It is well known that, at the foot of an obstacle, the boundary layer detaches from the 
bottom wall and generates a so-called "horseshoe vortex." Mignot and Riviere (2010) measured the detachment length of this horseshoe vortex and revealed that it is actually of the same order of magnitude as the detachment length of the hydraulic jump, even if remaining of smaller magnitude. This horseshoe vortex is expected to strongly impact the flow condition in the near-obstacle region and thus to affect the flow conditions in the region downstream from the hydraulic jump (at least in the region in front of the obstacle) and to strongly affect the conjugated water depths. The horseshoe vortex is finally expected to play a major role in the detachment length of the jump upstream from the obstacle. It should be reminded that the model of Moeckel (1949) was devoted to a flow configuration with no bottom wall, thus no boundary layer and thus no horseshoe-vortex. The horseshoe vortex in the measured configuration could be the cause for the discrepancies in Fig. 2.

To summarize, PR1 corresponds to a difference of definition between the measured and predicted detachment lengths; PR2 rather corresponds to an oversimplification of the model compared to the actual flow configuration. In order to identify which of these two potential reasons is/are responsible for the observed discrepancies, two complementary research studies were undertaken:

- A novel experimental approach, described in Sec. III, aims at verifying PR2 by measuring detachment lengths in front of obstacles after suppressing the horseshoe vortex. The principle is to remove the boundary layer by setting the obstacle in motion in water at rest, in a towing tank. The differences between the detachment lengths acquired in these conditions and those from Mignot and Riviere (2010) should be attributed only to the absence/presence of the horseshoe vortex.

- The numerical approach in Sec. IV aims at verifying at the same time PR1 and PR2, by computing numerically a supercritical open-channel flow skirting an obstacle (as for Mignot and Riviere, 2010) but by removing the boundary layer and subsequent horseshoe-vortex setting a free-slip condition on the bottom wall. The easy access to the numerical data should then permit to quantify both detachment lengths: that of the toe of hydraulic jump and of the flow regime transition.

\section{NOVEL EXPERIMENTAL APPROACH IN A TOWING TANK}

The aim of the novel experimental configuration is to obtain a detached hydraulic jump similar to that observed in front of mounted and emergent obstacles in supercritical flows (as for Mignot and Riviere, 2010) but in the absence of the horseshoe vortex. The strategy is to suppress the boundary layer of the inflow by considering an obstacle in motion, sliding on the bed and emerging from the free-surface, within a water basin at rest.

\section{A. Experimental setup}

The experiments are performed in the $35 \mathrm{~m}$ long, $0.9 \mathrm{~m}$ wide, and $1.2 \mathrm{~m}$ high towing tank of the LOMC, at the University of Le
Havre Normandie (France). In the measurement region, three horizontal plates of $3 \mathrm{~m}$ long each, made of black polyvinyl chloride (PVC), are laid one next to the other on the flume's bottom in order to reduce the roughness of the bed. The obstacles are translated horizontally by a carriage driven by a $600 \mathrm{~V}$ AC motor, located above the flume, at a selected velocity in water at rest with a constant depth $h_{1}$ (see Fig. 3). For each test, the water depth $h_{1}$ is measured with a micrometer gauge at a fixed location.

The obstacles are made of several 2 or $10 \mathrm{~mm}$ thick rectangular plates of PVC fixed together with a rod, to be able to regularly increase the obstacle width $R$. A thin wipe is fixed beneath the obstacle to reduce friction, and a weight is added above in order to ensure a permanent contact of the lower obstacle face with the bed. A vertical mast is finally rigidly fixed to the carriage. To limit the transmission of the carriage vibrations to the obstacle, the latter is attached to the mast through two $0.715 \mathrm{~m}$ long flexible transparent rods.

For each measurement, an acceleration protocol is established: the velocity is increased linearly over a $1 \mathrm{~m}$ long reach, the obstacle is then translated at a constant speed $U_{1}$ over $7 \mathrm{~m}$, and the velocity is decreased linearly over the last $1 \mathrm{~m}$. As the obstacle moves with constant velocity, the free-surface deformation in front of the obstacle is recorded from the top by using a $1280 \times 720$ px Nikon camera at $24 \mathrm{fps}$ with a $28 \mathrm{~mm}$ lens. The camera is fixed on the carriage at about $1.6 \mathrm{~m}$ above the obstacles such that the spatial resolution equals about $1.6 \mathrm{px} / \mathrm{mm}$. Given the negligible image distortion, the exact image calibration is performed for each test by clicking on the upstream obstacle edges, thus detecting the width of the obstacle in pixels. A mirror is additionally placed at $45^{\circ}$ on the side of the visualization zone and attached to the obstacle, in order to observe the vertical section of the hydraulic jump (see top left region of both photographs in Fig. 4).

\section{B. Parameter range}

The range of investigated parameters is strongly limited by the width of the flume, the conditions of occurrence of the detached hydraulic jump and the carriage maximum velocity. Indeed, for a Froude number $\mathrm{Fr}_{1}<3$, the toe of the hydraulic jump reaches the side walls of the tank on the side of the obstacle (rather than downstream) and propagates upstream leading to a straight jump in front of the obstacle; this process was explained in detail by Defina and Susin (2006). The Froude number is thus taken larger than 3 for all experiments (see Table I). Besides, the non-dimensional water depth $h_{1} / R$ has to be kept small enough to prevent any wall-jet-like bow

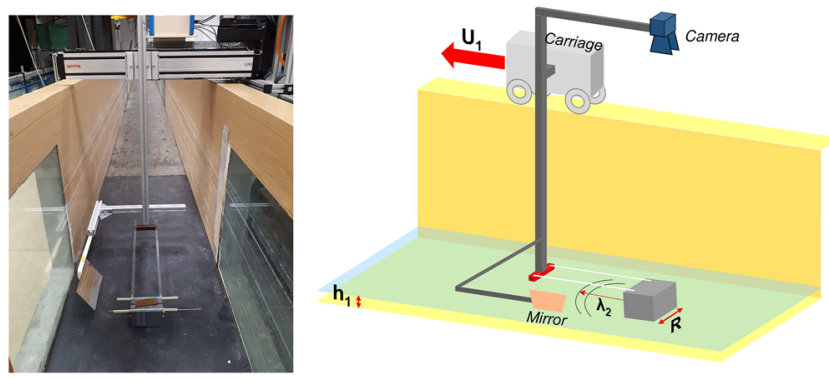

FIG. 3. Photograph and sketch of LOMC towing tank experiments with the obstacle moving at constant velocity in water at rest. 


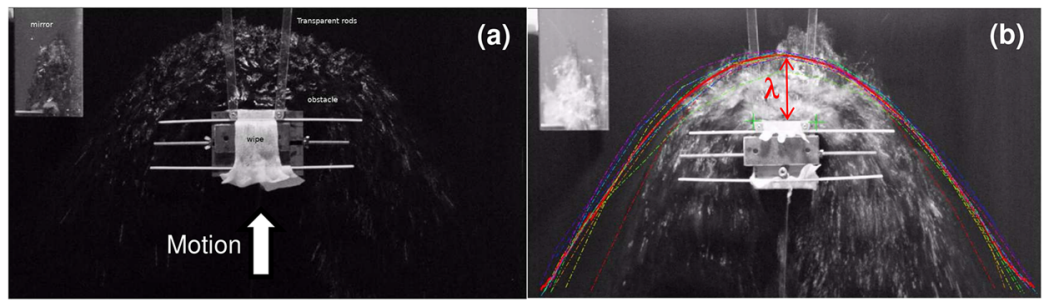

FIG. 4. Towing tank: photograph of the free-surface deformation facing the obstacle (a) and hydraulic jump detection method applied at 15 different times (colored dashed lines) (b). The top-left part of the image shows a side view of the hydraulic jump through the mirror.

TABLE I. Experimental series measured with the moving obstacle in water at rest in a towing tank (bold text refers to range of the studied parameter).

\begin{tabular}{lcccccc}
\hline \hline & $h_{1}(\mathrm{~mm})$ & $U_{1}(\mathrm{~m} / \mathrm{s})$ & $R(\mathrm{~mm})$ & $\mathrm{Fr}_{1}$ & $h_{1} / R$ & $\mathrm{Re}_{\mathrm{h}}$ \\
\hline Series 1 & $9.4-14.3$ & $1.06-1.31$ & $94-143$ & 3.5 & 0.1 & $\mathbf{4 0 ~ 0 0 0 - 7 5 ~ 0 0 0}$ \\
Series 2 & 12.3 & 1.22 & $88-176$ & 3.5 & $\mathbf{0 . 0 7 - 0 . 1 4}$ & 60000 \\
Series 3 & 6.45 & $0.75-1.51$ & 142 & $\mathbf{3 - 6}$ & 0.045 & $19470-35690$ \\
\hline \hline
\end{tabular}

wave, described by Riviere et al. (2017), from occurring instead of a detached hydraulic jump. Moreover, in all the experiments, the blockage ratio between the obstacle dimension $R$ and the width of the tank is kept smaller than 0.2 .

Given these constraints, three series of experiments are performed and detailed in Table I: (i) series 1 at constant nondimensional water depth and Froude number and varying Reynolds number, (ii) series 2 at constant Froude and Reynolds numbers and varying non-dimensional water depth, and (iii) series 3 at constant non-dimensional water depth and varying Froude number (also varying the Reynolds number for experimental limitation purposes, after verifying in series 1 that the Reynolds number hardly affects the detachment lengths, see further below).

\section{Detachment length measurement techniques}

Although the constant carriage velocity regime is reached at the end of the acceleration phase, some oscillations of the hydraulic jump detachment length and elevation are observed. These are partly due (i) to the vibrations of the carriage and slight variations in the bed elevation as the obstacle is translated and (ii) partly to the intrinsic oscillations of the detached hydraulic jump, already reported by Mignot and Riviere (2010). Thus, for each test, 15 non-consecutive images of the same experiment are selected throughout the recording process [Fig. 4(b)]. For each image, the position of the hydraulic jump is detected using a computer-aided manual detection method, similar to the one used by Mignot and Riviere (2010). This optical technique is validated with manual measurements of detachment lengths performed in the symmetry plane along the flume axis. The detachment length is then defined as the average over the 15 measurements of the distance from the obstacle upstream face to the toe of the hydraulic jump in the streamwise symmetry plane [Fig. 4(b)].

The uncertainty on the water depth $h_{1}$ is estimated to be $\pm 2 \mathrm{~mm}$ due to the non-perfect bottom topography. The uncertainty on the velocity is estimated to be $1 \mathrm{~mm} / \mathrm{min}$. Finally, the uncertainty on the detachment length is estimated as the root mean square of 15 independent measurements and is included in the graphs in Sec. V, leading to a maximum error estimate of $7 \%$.

\section{NUMERICAL CALCULATIONS}

The present section is devoted to evaluate PR1 and PR2 by means of numerical calculations which offer the possibility, at the same time, to remove the horseshoe-vortex (PR2) and to evaluate the difference in detachment length based on both definitions (PR1): the toe of the hydraulic jump (noted $\lambda_{2}$ ) and the location of flow regime transition (noted $\left.\lambda_{1}\right)$.

\section{A. Numerical method}

The numerical domain is slightly simplified in the sense that only half of it is computed, on one side of the inflow symmetry plane (see Fig. 5). Moreover, the obstacle length (along $x$ ) is extended up to the downstream boundary (to avoid computing a complex and costly wake downstream the obstacle). These simplifications do not introduce any specific error or uncertainty regarding the detachment length of the hydraulic jump. The 3D UnsteadyReynolds-Averaged-Navier-Stokes (URANS) equations are solved

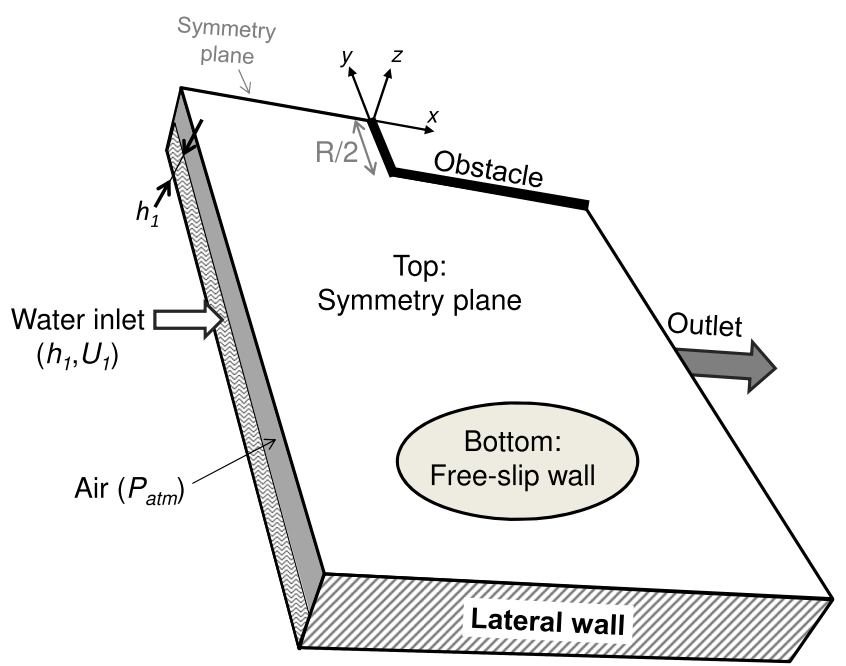

FIG. 5. Sketch of the numerical domain and boundary conditions. 


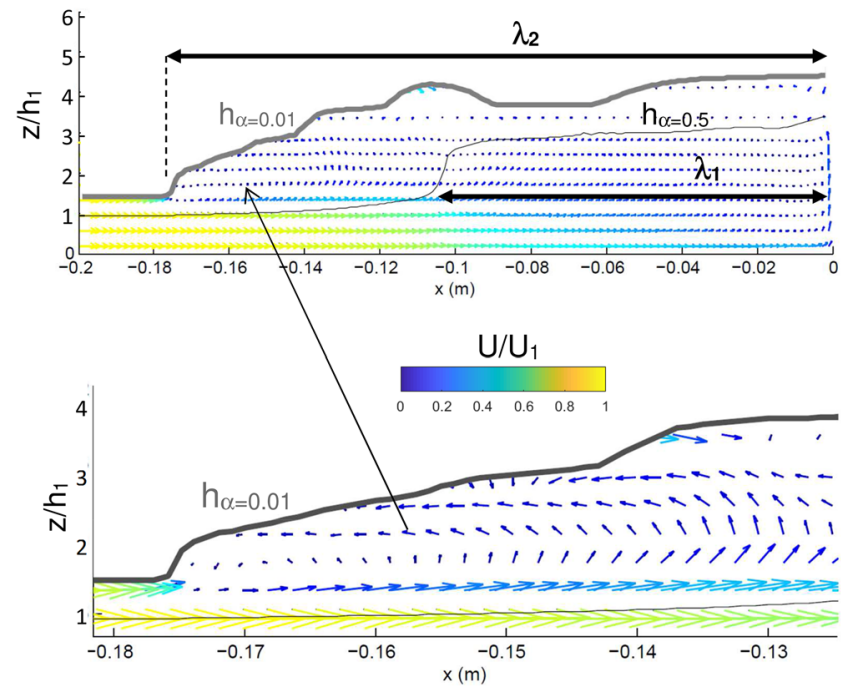

FIG. 6. Velocity field in the water volume (arrows, which color stands for velocity magnitude) along the symmetry plane $(y=0)$ for the configuration with $\mathrm{Fr}_{1}=2.5$ and $h_{1} / R=0.035$. The plain lines correspond to the free-surfaces, estimated as $h_{\alpha=0.5}$ (thin black line) and as $h_{\alpha=0.01}$ (thick gray line).

with a constant time step of $0.01 \mathrm{~s}$, using a typical k- $\varepsilon$ turbulence model and the Volume of Fluid (VOF) method to estimate the location of the air/water interface (i.e., the free-surface). Along the inlet boundary, a fixed water depth $h_{1}$ and a uniform streamwise velocity $U_{1}$ are imposed as boundary conditions. The obstacle and lateral boundaries are walls, the top surface is a plane of symmetry, the outlet considers a hydrostatic pressure distribution, and finally the bottom wall is set with free-slip conditions to avoid any boundary layer development and avoid the horseshoe vortex at the foot of the obstacle.

The mesh is made of about 350000 parallelepiped cells aligned with the walls and axes directions, refined along the $z$ axis in the water volume (lower region), with cell sizes equal to $\Delta \mathrm{x}{ }^{*} \Delta \mathrm{y}{ }^{*} \Delta \mathrm{z}$ $\approx 7.5 * 7.5 * 1.8 \mathrm{~mm}$. To verify the dependency of the computed flow pattern to the grid size, some calculations were repeated using a mesh comprising 900000 cells, refined in the water volume with $\Delta \mathrm{z}=1 \mathrm{~mm}$. The difference in detachment length using this refined mesh remained limited to about $3 \%$.

Apart from the $3 \mathrm{D}$ velocity and Reynolds stress component fields computed by the numerical model, the VOF method estimates the ratio between water and total volume (noted $\alpha$ ) in each cell $i$ : $\alpha_{i}=V_{\text {water }} /\left(V_{\text {water }}+V_{\text {air }}\right)$. Considering a small (respectively high) a threshold value permits to identify the regions where the water/air mixture is mainly composed of air (respectively water). Following analysis considers two distinct threshold values (see Fig. 6): $a=0.01$ represents the location of the highly aerated air/water mixture with only $1 \%$ of water and $99 \%$ of air (corresponding water depth is noted $h_{\alpha=0.01}$ ); $\alpha=0.5$ represents the usual free-surface (i.e., mean interface) between both phases (corresponding water depth is noted $h_{\alpha=0.5}$ ).

\section{B. Definition of computed detachment lengths}

Figure 7 reveals that the horizontal water depth field follows a typical hyperbolic curve wrapping around the obstacle, in agreement with measurements from Mignot and Riviere (2010). The approaching streamlines start deflecting from their initial axis (along $x$ ) when crossing the hydraulic jump, where the velocity magnitude suddenly decreases and the water depth $h_{\alpha=0.5}$ suddenly increases. From upstream to downstream, $h_{\alpha=0.01}$ and $h_{\alpha=0.5}$ exhibit different behaviors (Fig. 6): $h_{\alpha=0.01}$ starts increasing at a distance noted $\lambda_{2}$ from the obstacle and then oscillates until reaching the obstacle, while $h_{\alpha=0.5}$ suddenly increases at a shorter distance from the obstacle, noted $\lambda_{1}$. In fact, $\lambda_{1}$ appears to be the location where the flow regime changes from super- to subcritical, i.e., where $\mathrm{Fr}=1$ (see Fig. 7). Figure 6 additionally reveals a flow recirculation in the air/water mixture just downstream from $\lambda_{2}$.

To summarize: $\lambda_{2}$ can be defined as the detachment length of the "toe of the hydraulic jump" where the air/water mixture starts to rise above $h=h_{1}$. On the other hand, $\lambda_{1}$ is defined as the detachment length of the sudden flow regime transition from $\mathrm{Fr}>1$ to $\mathrm{Fr}<1$, somehow hidden from optical access in the experiments by the air/water mixture. The relative uncertainty regarding these two detachment lengths is estimated, through estimations by different experts, to about $2 \%$ during the post-processing of the computed water depth field.
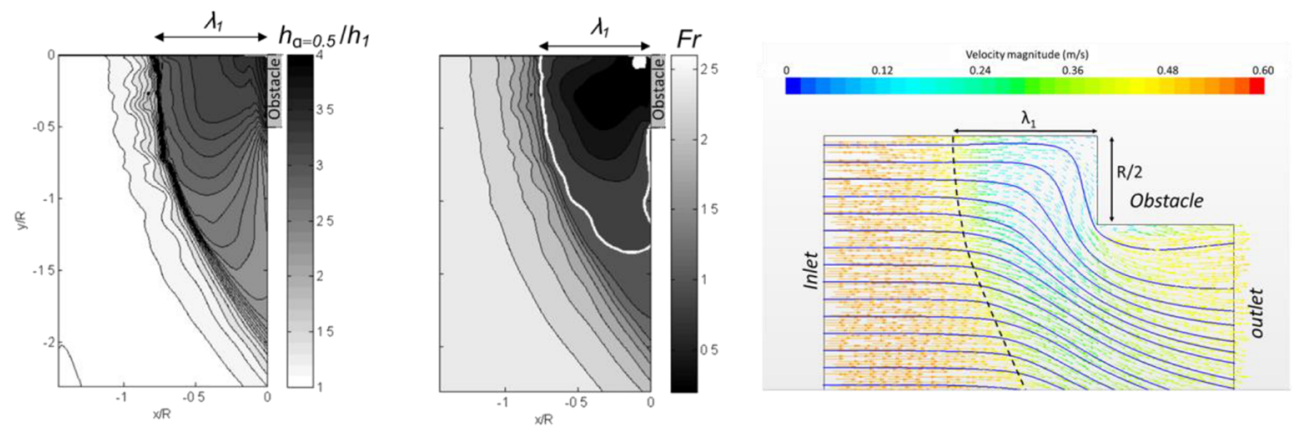

FIG. 7. Computed normalized water depth $\left(h_{\alpha=0.5} / h_{1}\right.$ obtained with $\alpha=0.5$, left), Froude number (Fr, center) upstream from the obstacle, and velocity field at $z=h_{1} / 2$ for the configuration $\mathrm{Fr}_{1}=2.5$ and $h_{1} / R=0.035$. The white line in the Froude number map corresponds to $\mathrm{Fr}=1$; in the velocity field map, the blue line are projections of streamlines and the dashed line is the location of the maximum $h_{\alpha=0.5}$ gradient. 
TABLE II. Computed flow series.

\begin{tabular}{lcccccc}
\hline \hline & $h_{1}(\mathrm{~mm})$ & $U_{1}(\mathrm{~m} / \mathrm{s})$ & $R(\mathrm{~mm})$ & $\mathrm{Fr}_{1}$ & $\mathrm{~h}_{1} / R$ & $\mathrm{Re}_{h}$ \\
\hline Series A & $4-5$ & $0.46-0.51$ & $120-140$ & 2.3 & $0.033-0.036$ & $\mathbf{7 3 0 0 - 1 0 2 0 0}$ \\
Series B & 4 & 0.516 & $60-160$ & 2.6 & $\mathbf{0 . 0 2 5 - 0 . 0 6 7}$ & 8200 \\
Series C & $4-4.7$ & $0.49-0.57$ & $120-130$ & $\mathbf{2 . 3 - 2 . 9}$ & 0.035 & $8500-10100$ \\
\hline \hline
\end{tabular}

\section{Computed flow series}

Numerical calculations are performed for three flow series by varying one of the non-dimensional parameter [Eq. (1)] at a time (Table II). Both detachment lengths $\left(\lambda_{1}\right.$ and $\left.\lambda_{2}\right)$ are extracted from each calculation. The main limit in the selection of these parameters is related to the flow configurations with high Froude numbers $\left(\mathrm{Fr}_{1}>3\right)$, for which the unsteadiness of the flow makes it very difficult and uncertain to estimate the time-averaged detachment lengths. The selected upstream Froude numbers are thus taken as high as possible below this limit, i.e., in the range 2.3-2.9; they remain lower than for the experiment by Mignot and Riviere (2010) and the novel towing tank experiment. The normalized upstream water depth is taken in the range of measurements by Mignot and Riviere (2010) and the towing tank measurements (i.e., 0.030.07). The Reynolds number is taken in the range of measurements by Mignot and Riviere (2010) but remains lower than that in the towing tank. Table II summarizes the flow patterns computed numerically.
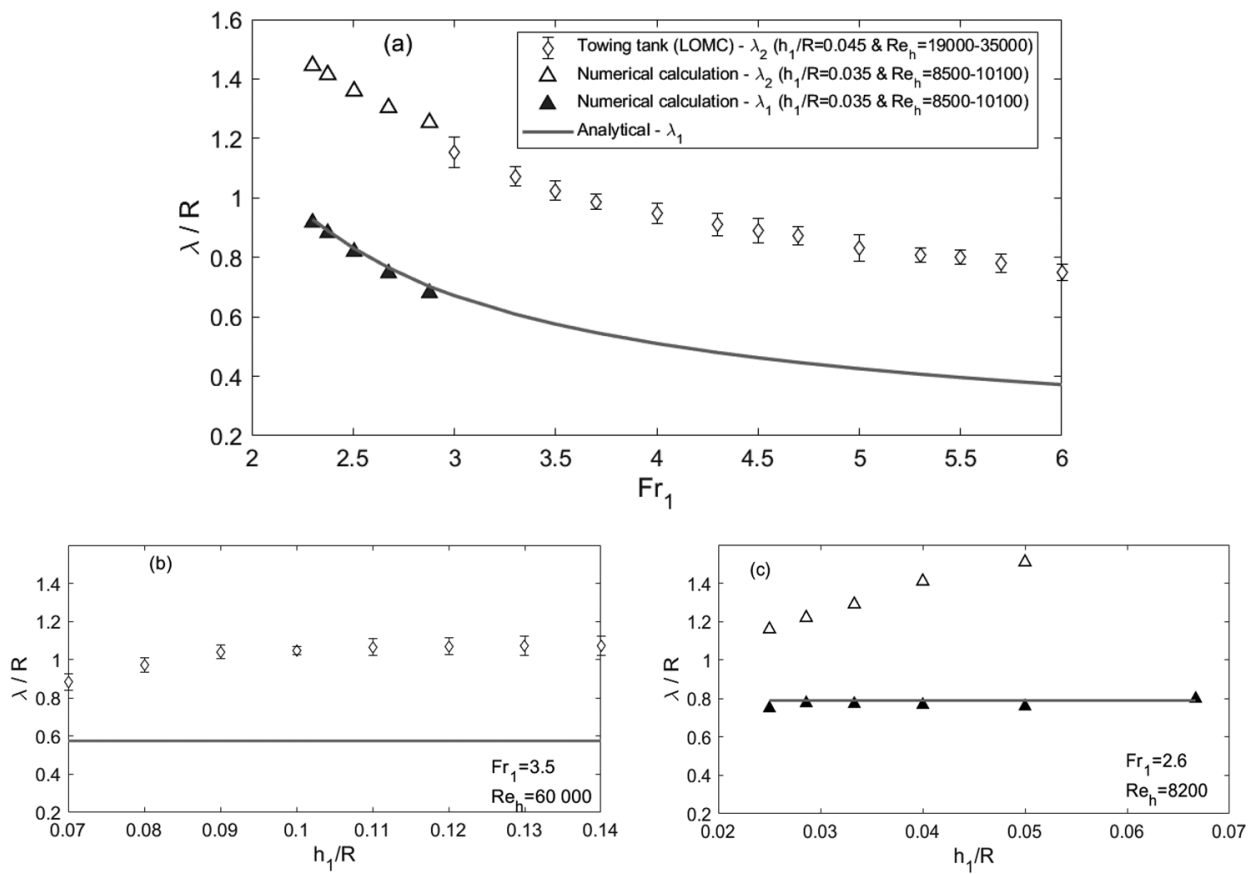

FIG. 8. Comparison of hydraulic jump detachment lengths $\lambda$ : measured in the towing tank (LOMC), numerically computed and predicted analytically, as a function of the inflow Froude number (a), of the normalized water depth $[(b)$ and (c)], and of the Reynolds number [(d) and (e)].
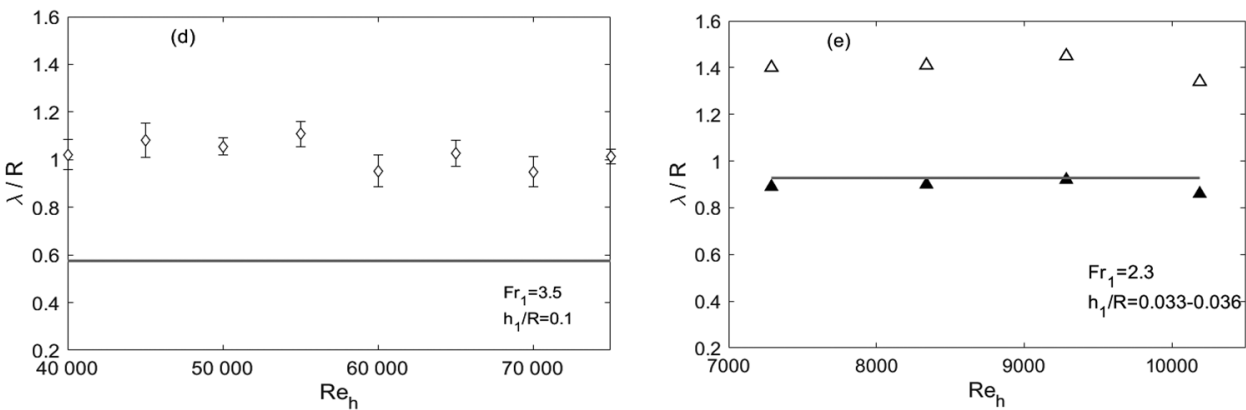

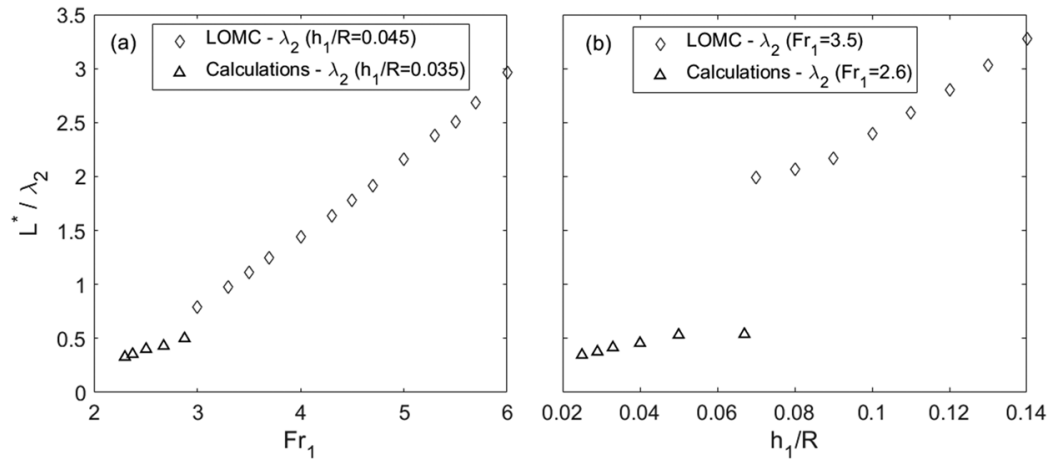

FIG. 9. Ratio between empirical length for ordinary straight hydraulic jumps $L^{*}$ and measured or computed detachment length $\lambda_{2}$ with similar upstream (supercritical) conditions as a function of the Froude number [(a) series $C$ from Table II and series 3 from Table I] and the normalized upstream depth [(b) series B from Table $\|$ and series 2 from Table I].

\section{RESULTS}

Figure 8 compares the detachment lengths of the hydraulic jump along the symmetry plane (i) measured in the towing tank (Table I), (ii) computed numerically (Table II), and (iii) predicted by the analytical model [Eq. (6)]. Unfortunately, as discussed above, the towing tank measurements could only be performed for flows with $\mathrm{Fr}_{1}>3$, while numerical calculations could only be performed for flows with $\mathrm{Fr}_{1}<3$. Consequently, the following analysis does not comprise any common configuration among the three approaches. Still, Figs. 8(a), 8(b), and 8(d) reveal that the detachment lengths measured in the towing tank in front of the moving obstacle strongly exceed the analytical values but fairly agree with the computed values corresponding to the toe of the hydraulic jump $\left(\lambda_{2}\right)$. Besides, Figs. $8(\mathrm{a}), 8(\mathrm{c})$, and $8(\mathrm{e})$ reveal that the computed detachment lengths corresponding to the flow regime transition $\left(\lambda_{1}\right)$ fairly agree with those predicted by the analytical model. This confirms the following:

- The detachment lengths measured in the towing tank correspond to that of the toe of the hydraulic jump. They are thus noted $\lambda_{2}$ in Fig. 8. They are in agreement with the computed $\lambda_{2}$ values.

- The analytical model [Eq. (6)] accurately predicts the flow regime transition detachment lengths from the super- to subcritical regime. These are thus noted $\lambda_{1}$ in Fig. 8. Remind that this is only valid in cases where no boundary layer, i.e., no horseshoe vortex, affects the development of the detached hydraulic jump.

- The detachment length of the toe of the hydraulic jump is strongly affected by the presence of a horseshoe vortex in front of the obstacle. Indeed, when comparing Fig. 2 (measured data-with a horseshoe vortex) and Figs. 8(a), 8(c), and $8(\mathrm{e})$ (computed $\lambda_{2}$ without a horseshoe vortex-with similar Froude number and $h_{1} / R$ ), the horseshoe vortex (in Fig. 2) strongly reduces this detachment length. As already mentioned by Mignot and Riviere (2010), it is expected that the horseshoe vortex acts as a forward facing step which modifies the subcritical backwater curve upstream from the obstacle and thus highly affects the conjugated depths of the hydraulic jump.

On the other hand, these results confirm that $\lambda_{1}$ (location of transition from the super- to subcritical regime) decreases with the inflow Froude number [Fig. 8(a)] but is unaffected by the normalized water depth [Fig. 8(c)] and Reynolds number [Fig. 8(e)]. This observation is in agreement with that of the shock wave detachment in aerodynamics (see Moeckel, 1949).

Figure 8(a) also reveals that $\lambda_{2}$ (the detachment length of the toe of the hydraulic jump) decreases with the Froude number, in a similar tendency as for $\lambda_{1}$, with a ratio $\lambda_{2} / \lambda_{1}$ ranging from 1.5 to 2. Moreover, $\lambda_{2}$ appears to be unaffected by the Reynolds number [Figs. 8(d) and 8(e)] but increases with the normalized incoming water depth [Figs. 8(b) and 8(c)]. This tendency is in fair agreement with the empirical length of ordinary straight hydraulic jumps (noted $L^{*}$ ) which, for a given upstream Froude number, linearly increases with the upstream water depth $h_{1}$ (Chow, 1959). However, Fig. 9 reveals that $\lambda_{2}$ can be lower or larger than $L^{*}$ with similar upstream conditions $\left(h_{1}, \mathrm{Fr}_{1}\right)$ with $L^{*} / \lambda_{2}$ increasing with both $\mathrm{Fr}_{1}$ and $h_{1} / R$. There exists a strong analogy of this ratio $L^{*} / \lambda_{2}$ with the ratio $L^{*} / L_{g}$ for an impact jump with $L_{g}$ being the distance from the toe of the hydraulic jump to the gate: Hager (1994) showed that this ratio governs the impact jump processes. Oppositely, in the present flow configurations with a mounted obstacle that the flow must skirt from the sides, no qualitative difference in the flow pattern can be observed as a function of $L^{*} / \lambda_{2}$.

\section{CONCLUSIONS}

The present work aimed at evaluating the capacities of an analytical model, initially applied to aerodynamics and adapted herein to hydraulics, to predict the detachment length of hydraulic jumps in front of mounted and emerging obstacles in supercritical openchannel flows. This work permitted to clearly distinguish between two different flow structures, characteristic of detached jumps: the classical toe of the hydraulic jump (which detachment length is noted $\lambda_{2}$ ) and the location of flow regime transition from the superto subcritical regime (with a shorter detachment length noted $\lambda_{1}$ ). The visible detachment length, frequently reported on experiments, corresponds to the toe of the hydraulic jump $\left(\lambda_{2}\right)$ where a vertical flow recirculation takes place at the free-surface. It thus differs from the detachment length predicted by the analytical model $\left(\lambda_{1}\right)$, which corresponds to the location of flow regime transition from the supercritical to subcritical regime, with a sudden water depth increase and velocity and Froude number decrease.

When comparing the measured and computed detachment lengths, the paper revealed that the present analytical model is 
unable to predict the detachment lengths of the toe of the hydraulic jump, in the presence or absence of a boundary layer (and thus of a horseshoe vortex in front of the obstacle). Additional work is then required before being able to predict such detachment length. Oppositely, the present work assesses that the analytical model predicts with high accuracy the location of transition from the super- to subcritical flow regime along the symmetry plane, in the absence of a boundary layer (and thus of a horseshoe vortex). Note, however, that the analytical model was validated against numerical calculations [Figs. 8(a), 8(c), and 8(e)] but could not be compared to measurements due to the lack of experimental data concerning the flow regime transition location. These data should be obtained by measuring the water depth field with ultrasonic probes and the velocity field within the hydraulic jump, using particle image velocimetry, laser Doppler anemometry (LDA), or more advanced measurement methods; they, however, remain quite challenging due to the air entrainment and limited optical access.

Finally, both hypotheses proposed above are valid: the analytical model fails at predicting the location of the toe of the hydraulic jump in a supercritical open-channel flow skirting a mounted and emerging impervious obstacle due both to the width of the hydraulic jump and the presence of the horseshoe vortex.

From an engineering point of view, the location of flow regime transition corresponds to a sudden velocity decrease, water depth increase, and Froude number decrease (below 1). This sudden transition strongly affects the flow characteristics in the water column as well as the shear stress at the bed. It is then expected to be of high interest for applications such as the drag force acting on the obstacle, the scour occurring at the toe of the obstacles below the hydraulic jump, and the capacity of fishes to go through steep fishways with emerging obstacles. Nevertheless, in its present form, the analytical model is not yet able to predict this location for configurations with a boundary layer and a horseshoe vortex.

\section{ACKNOWLEDGMENTS}

The authors would like to thank the GIS-HED ${ }^{2}$ (French scientific interest group: Hydraulics for Environment and Sustainable Development, (http://wikhydro.developpement-durable.gouv.fr/ index.php/GIS_HED\%C2\%B2) for the financial support for this project, including the scholarship of first author's master thesis.

\section{REFERENCES}

Cassan, L., Tien, T. D., Courret, D., Laurens, P., and Dartus, D., "Hydraulic resistance of emergent macroroughness at large Froude numbers: Design of nature-like fishpasses," J. Hydraul. Eng. 140(9), 04014043 (2014).

Chow, V. T., Open Channel Hydraulics (McGraw-Hill, New York, 1959).

De Chant, L. J. and Caton, J. A., "Measurement of confined supersonic, 2-d jet lengths using the hydraulic analogy," Exp. Fluids 24(1), 58-65 (1998).

Defina, A. and Susin, F. M., "Multiple states in open channel flow, in vorticity and turbulence effects in fluid structures interactions," in Advances in Fluid Mechanics, edited by Brocchini, M. and Trivellato, F. (WIT Press, Southampton, UK, 2006), pp. 105-130.

Hager, W. H., "Impact hydraulic jump," J. Hydrol. Eng. 120, 633-637 (1994).

Ippen, A. T., "Mechanics of supercritical flow: 1st paper of high velocity flow in open channels: A symposium," Trans. ASCE 116, 268-295 (1951).

Jiang, Q. and Smith, R. B., "V-waves, bow shocks, and wakes in supercritical hydrostatic flows," J. Fluid Mech. 406, 27-53 (2000).

Lukkunaprasit, P., Ruangrassamee, A., and Thanasisathit, N., "Tsunami loading on buildings with openings", Sci. Tsunami Hazards 28(5), 303-310 (2009).

Mignot, E., Moyne, T., Doppler, D., and Riviere, N., "Clear-water scouring process in a flow in supercritical regime," J. Hydraul. Eng. 142(4), 04015063 (2016).

Mignot, E., Paquier, A., and Haider, S., "Modeling floods in a dense urban area using 2D shallow water equations," J. Hydrol, 327(1-2), 186-199 (2006).

Mignot, E. and Riviere, N., "Bow-wave-like hydraulic jump and horseshoe vortex around an obstacle in a supercritical open channel flow," Phys. Fluids 22, 117105 (2010).

Misra, S., Kirby, J., Brocchini, M., Veron, F., Thomas, M., and Kambhamettu, C., "The mean and turbulent flow structure of a weak hydraulic jump," Phys. Fluids 20, 035106 (2008).

Moeckel, W. E., “Approximate method for predicting form and location of detached shock waves ahead of planar or axially symmetric bodies," Technical Note 1921, National Advisory Committee for Aeronautics, 1949.

Riviere, N., Vouillat, G., Launay, G., and Mignot, E., "Emerging obstacles in supercritical open-channel flows: Detached hydraulic jump versus wall-jet-like bow wave," J. Hydraul. Eng. 143(7), 04017011 (2017).

Sturm, M., Gems, B., Keller, F., Mazzorana, B., Fuchs, S., Papathoma-Köhle, M., and Aufleger, M. "Experimental analyses of impact forces on buildings exposed to fluvial hazards," J. Hydrol. 565, 1-13 (2018).

Witt, A., Gulliver, J. S., and Shen, L., "Numerical investigation of vorticity and bubble clustering in an air entraining hydraulic jump," Comput. Fluids 172, 162-180 (2018)

Wüthrich, D., Pfister, M., Nistor, I., and Schleiss, A. J., "Experimental study on the hydrodynamic impact of tsunami-like waves against impervious free-standing buildings," Coastal Eng. J. 60(2), 180-199 (2018). 